\title{
Differential Management Strategies and Outcomes in Patients over 65 years with Acute Calculous Biliary Disease-Results and Insights from the ESTES Complicated Biliary Calculous Disease Snapshot Audit
}

Gary Alan Bass ( $\nabla$ garybassmd@gmail.com )

Orebro Universitet https://orcid.org/0000-0002-1918-9443

Amy E. Gillis

Tallaght University Hospital

\section{Yang Cao}

Orebro Universitet

Shahin Mohseni

Orebro Universitet

ESTES Cohort Studies Collaborative Group

ESTES

\section{Research article}

Keywords: cholecystitis, elderly, cholecystectomy, morbidity, emergency surgery

Posted Date: June 18th, 2020

DOI: https://doi.org/10.21203/rs.3.rs-23736/v1

License: (c) (i) This work is licensed under a Creative Commons Attribution 4.0 International License. Read Full License 


\section{Abstract}

Background: Acute complicated calculous biliary disease (ACCBD) may pose challenges in an ageing population. Frailty and comorbidities increase the potential risks of surgery; thus, surgeons may opt to offer operative treatments less often to their older patients. We set out to capture the incidence and treatment algorithms used across Europe to treat older patients presenting with ACCBD.

Methods: Analysis of the European Society of Trauma and Emergency Surgery (ESTES) 2018 Acute Complicated Calculous Biliary Disease audit was performed. Patients undergoing emergency hospital admission with ACCBD between 1 October 2018 and 31 October 2018 were included. The primary outcome measure was operative intervention in patients over and under 65 years of age. Mortalities, postoperative morbidity, time to operative intervention, post-acute disposition and length of hospital stay were measured as secondary outcomes.

Results: The median age of the 338 patients admitted to the snapshot was 67 years; 185 patients $(54.7 \%)$ were over 65 years at time of admission. Significantly fewer patients over 65 underwent definitive surgical treatment, compared with those under $65(37.8 \%$ vs. $64.7 \%, p<0.001)$. Surgical complications were seen more frequently in the over 65 cohort. Post-operative mortality was seen in $2.2 \%$ of over $65 \mathrm{~s}$ versus $0.7 \%$ under $65(p=0.253)$. Mean post-operative length of stay was significantly longer in the elderly cohort. In patients surviving to discharge, post-acute convalescence or rehabilitation was required in $13.3 \%$ in the elderly cohort versus $1.9 \%$ of those under $65(p=0.002)$.

Conclusions: Elderly patients commonly present with ACCBD. Increased frailty and incidence of comorbid disease in this population increases the potential surgical risk. In our snapshot, elderly patients represented the majority, but far fewer were offered definitive surgical treatment. Post-operative mortality, morbidity, length of post-operative in-hospital stay and the requirement for post-discharge convalescence were higher in this group.

\section{Background}

Over the last 25 years, the number of older people undergoing emergency non-cardiac surgical procedures has increased faster than the rate of population ageing. Despite advances in peri-operative care, and changing patient expectations, age and pre-existing comorbidities remain the main predictors of adverse postoperative outcomes in the older surgical population[1-8]. In particular, gallbladder surgery has been highlighted as accruing excess morbidity and mortality in the elderly patient[9]. The role of frailty as an independent risk factor for adverse postoperative outcomes is also now emerging[10-13]. An ageadjusted Charlson co-morbidity index[14-16] has been recently validated, while limitations in the discriminating power of the prognostic p-POSSUM score have been recognised in the older patient[17].

Acknowledging that acute complicated calculous biliary disease is a common set of clinical problems which presents frequently to general surgeons, the Cohort Studies Group of the European Society for Trauma and Emergency Surgery set out to capture real-world data on the epidemiology and contemporary 
management of these patients[18, 19]. While traditional medical school teaching identifies the 6Fs (fair, fat, fertile, female, forty and family history) as predictors of the population most likely to present with symptomatic gallstone disease[20], it was notable that the cohort presenting to European emergency departments were older, with a median age of 67 years.

We aimed to assess patterns of surgical practice regarding these older patients as well as collating data on how outcomes differ in this cohort compared with younger patients.

\section{Methods}

\section{Protocol}

This prospective, observational, multi-centre audit was conducted in line with a pre-specified protocol which was registered with ClinicalTrials.gov (Trial \# NCT03610308).

\section{Centre eligibility}

Any unit undertaking adult acute care surgery was eligible to register to enter patients into the study. No minimum case volume, or centre-specific limitations were applied. The study protocol was disseminated to registered members of the European Society of Trauma and Emergency Surgery (ESTES), and through national surgical societies.

\section{Patient eligibility}

Adult patients (over 18 years of age) admitted for acute gangrenous or perforated calculous cholecystitis (AAST Severity Grade II or above), choledocholithiasis or complications of cholelithiasis and/or choledocholithiasis or biliary pancreatitis. Procedures performed on these patients included cholecystectomy (open, laparoscopic or robotic), choledochotomy/common bile duct exploration (open, laparoscopic or robotic), pancreatic necrosectomy, gastrojejunostomy, cyst gastrostomy, endoscopic retrograde choledochopancreatography (ERCP) or endoscopic ultrasound (EUS), percutaneous cholecystostomy (transhepatic or transperitoneal), percutaneous transhepatic drainage, stone removal or stent placement. Patients with uncomplicated biliary colic, biliary dyskinesia or uncomplicated acute calculous cholecystitis (AAST Grade I) were excluded from the study.

\section{Data capture}

Data were recorded contemporaneously and stored on a secure, user-encrypted online platform $\left(\right.$ REDCap $\left.^{\circledR}\right)$ without patient-identifiable information. Centres were asked to validate that all eligible patients during the study period had been entered, and to attain $>95 \%$ completeness of data field entry prior to final submission. Quality assurance mentorship was provided by at least one consultant/attending-level surgeon at every participating site. 
Outcomes were compared between patients aged over and under 65 years. The primary outcome measure was index admission surgical definitive treatment (cholecystectomy). The secondary outcome measures were length of stay, the postoperative major complication rate defined as Clavien-Dindo classification grade 3 to 5 (reoperation, reintervention, unplanned admission to intensive care unit, organ support requirement or death), the postoperative length of stay (in whole days), with day of surgery as day zero, and the postoperative mortality rate, defined as death within 30 days of surgery.

\section{Statistical analysis}

The descriptive and inferential statistical analyses were performed using Stata 15.1 (StataCorp LLC, College Station, TX, USA) and the jamovi project (www.jamovi.com, 2019) utilizing the R language for statistical computing. Effect estimates are presented as odds ratios (OR) with 95\% confidence intervals and two-tailed P-values. An alpha significance level of 0.05 was used through-out. Measures of central tendency were presented as mean ( \pm standard deviation; median, interquartile range).

\section{Ethical considerations}

All 25 participating centres (see Supplemental Table of Collaborators) provided local institutional review board approval or equivalent as a requirement of registration. No patient consent was sought since the current study was purely observational and did not change the medical course of any patient. All data were irrevocably de-identified at source when uploaded to the secure study database.

\section{Results}

\section{Participating Centres}

Following an open call for participation by ESTES in May 2018, 38 centres expressed interest in participating. Of those, 25 centres completed the local ethics approval process and proceeded to enrol patients to the study. These centres came from 9 countries (Austria, Italy, Ireland, Romania, Spain, Sweden, Portugal, United Kingdom, and United States of America).

\section{Patient Demographics}

Three-hundred and thirty-eight individual patients admitted between $1^{\text {st }}$ October and $31^{\text {st }}$ October 2018 were enrolled in the study and followed up until 60 days post-admission (latest patient $31^{\text {st }}$ December 2018). Female patients outnumbered male $(53.8 \%$ vs. $46.2 \%)$ in the cohort as a whole. The median age at time of diagnosis was 67 years (mean \pm sd; range: $65 \pm 18$ years; $18-100)$. One hundred eighty five (54.7\%) of patients were over the age of 65 years at time of index admission, equal gender balance was seen in patients over 65 , with $50.8 \%$ male and $49.2 \%$ being female. The median body mass index (BMI) was calculated as $27.34 \mathrm{~kg}(28.51 \pm 6.14 \mathrm{~kg})$. Patients who currently smoke tobacco products accounted for $55 / 338(16.3 \%$ ), while there were $118 / 338(34.9 \%$ ) ex-smokers (>6 weeks prior to admission) and $165 / 338(48.8 \%)$ patients who had never smoked; just $8 \%$ of patients over 65 years currently smoked, 
compared with $26.1 \%$ of those under $65(\mathrm{p}=0.002)$. Patients over 65 years were significantly more likely to have a history of ischemic heart disease $(0.4 \%$ vs $10.4 \%, p=0.040)$, congestive heart failure $(19.5 \%$ vs. $0.03 \%, \mathrm{p}<0.001)$, cerebrovascular disease $(18.4 \%$ vs. $0.05 \%, \mathrm{p}<0.001)$, insulin-dependent diabetes mellitus ( $41 \%$ vs. $13 \%, p=0.003)$ or chronic renal disease $(10 \%$ vs. $0.02 \%, p=0.003)$ than patients under 65 . No difference was seen in previous history of abdominal surgery between patients over 65 years (34\%) and those under $65(37 \%), p=0.487$. As a consequence, the mean( \pm sd; median,IQR) age-adjusted Charlson Comorbidity Index (aaCCl) was significantly higher in patients over 65 than in those under $65(8.51 \pm 2.37$; $8,7-10$ vs. $2.41 \pm 2.31 ; 2,0-4, p<0.001)$. Significantly more patients over 65 were scored as ASA Grade $2-4$ compared with patients under 65 (93\% vs. $64 \%$; $p<0.001)$. However, while the aaCCl was significantly higher in patients over 65 managed non-operatively than in those undergoing surgical intervention $(8.79 \pm 2.44 ; 8,7-10$ vs. $8.04 \pm 2.18 ; 8,6-9, p=0.027)$, it is questionable if this represents a clinicallymeaningful difference.

\section{Diagnosis}

Acute calculous cholecystitis was present in $45.9 \%$ patients, acute biliary pancreatitis in $20.7 \%$, choledocholithiasis in $31.9 \%$ of whom $43.5 \%$ had cholangitis. Five patients $(1.5 \%)$ were admitted for treatment of Mirizzi syndrome or bilioenteric fistula. Acute biliary pancreatitis was also graded using the AAST Emergency Surgery grading system. Of total 70 patients with diagnosis of pancreatitis, the majority had interstitial pancreatitis (95.7\%), and 3 (4.3\%) suffered from necrotising pancreatitis.

\section{Operative Management}

Of the 338 patients enrolled in the study, $50 \%$ underwent surgical intervention, while $50 \%$ had not received operative treatment by the end of the 60-day follow-up period. One hundred and fifty-two patients (89.9\%) underwent surgical intervention during the index admission, while a further seventeen (10.1\%) were operated upon after discharge from index admission but prior to the closure of the study database (with a mean interval to cholecystectomy from index admission being $56 \pm 25$ days (range 13-97).

Cholecystectomy was performed in $99.3 \%$ cases; the only other operation performed was pancreatic necrosectomy (including splenic flexure colonic resection) in one patient $(0.7 \%)$. Laparoscopic cholecystectomy was completed in $83.4 \%$ of the operated cases, including four laparoscopic sub-total cholecystectomies. Conversion to open cholecystectomy occurred in thirteen (8.6\%) cases, five in patients under 65 and eight in those over 65 . A further twelve (7.9\%) cholecystectomies were performed as open from the beginning of the procedure - eight in patients under 65 years and six in patients over 65 .

During the index admission, the mean ( \pm sd; median,IQR) time from admission to operation was significantly-shorter in older patients (7.25 $\pm 19.4 ; 1,0-3.5$ days) than in patients under 65years (10.4 $\pm 22 ; 2,1-6.25$ days, $p=0.039)$. However, a significantly-lower number of patients over 65 years were offered definitive surgical treatment, with $37.5 \%$ operated, compared with $64.7 \%$ under 65 years, $p<0.001$. Mean ( \pm sd; median,IQR) total length of in-patient stay (irrespective of diagnosis) did not differ between groups, at $9.56( \pm 8.25 ; 7,5-12)$ days for patients over 65 years vs. $8.85( \pm 10.41 ; 7,4-9)$ days for those under 
65 years $(\mathrm{p}=0.483)$; however, post-operative length of hospital stay was significantly longer in patients over 65 at $8.65( \pm 9.33 ; 5,3.75-9.25)$ days vs $4.78( \pm 6.01 ; 3,2-6)$ days $(p=0.002)$, (Figure 3 ).

\section{Endoscopic Management}

Endoscopic evaluation and management of the common bile duct was undertaken in $29 \%$ of patients $34.1 \%$ in patients over 65 and $22.9 \%$ in patients under $65(p=0.095)$. Of these, Endoscopic Retrograde Cholangiopancreatography (ERCP) with duct clearance and sphincterotomy was performed in 76 (77.6\%), ERCP and stent placement in 19 (19.4\%) and EUS alone, without therapeutic duct management, was performed in three $(3.1 \%)$ cases. Of those patients undergoing ERCP, nine $(9.2 \%)$ patients experienced complications - post-ERCP pancreatitis in six (6.1\%), bleeding in three (3.1\%); no procedure was complicated by perforation. Mean ( \pm sd; median,IQR) time from admission to endoscopy was significantly longer in patients over the age of 65 years $(9.27 \pm 11.2 ; 6.5,2.25-11$ days) compared with patients under 65 $(5.00 \pm 6.71 ; 4,2-5$ days, $p=0.009)$, (Figure 3$)$.

\section{Interventional Radiologic Management}

Interventional radiologic management of the gallbladder or common bile duct was undertaken in $7.7 \%$ $(n=26)$ of patients -11 patients under the age of 65 years and 15 patients over $65(p=0.830)$. Cholecystostomy was performed in $23(88.5 \%)$ of these cases, percutaneous radiologic drainage of a collection or abscess was performed in one (3.8\%) patient and percutaneous transhepatic cholangiography was performed in two (7.7\%). No complication was recorded for patients undergoing interventional radiologic procedures. Mean ( \pm sd; median,IQR) time from admission to endoscopy did not differ between patients over the age of 65 years $(4.11 \pm 12.3 ; 2,0.25-7$ days) compared with patients under 65 (3.65 $\pm 4.33 ; 2,0-6$ days), $p=0.738$, (Figure 3 ).

\section{Morbidity and Mortality}

Five deaths were recorded (1.4\%) in the total cohort- one (1.4\%) patient in those with suffering gallstone pancreatitis, two (4.2\%) in patients with cholangitis and two (1.2\%) patients with acute cholecystitis, both of which had an AAST Grade IV cholecystitis. Only one (0.58\%) post-operative mortality was recorded, following cholecystectomy for AAST Grade IV cholecystitis. Four deaths occurred in patients over 65 $(2.2 \%)$, compared with one $(0.7 \%)$ under 65 years $(p=0.253)$. Of the 333 patients surviving to discharge, significantly more patients over 65 years had ongoing morbidity requiring post-acute convalescence or rehabilitation $(13.3 \%)$, compared with $(1.9 \%)$ under the age of $65(p=0.002)$.

Post-operative complications were seen more commonly in patients over 65 compared with younger patients (18.5\% vs $9 \%, p=0.102)$; although it did not achieve statistical significance due to a low incidence, bile duct injury and haemorrhage requiring return to the OR were more common in the elderly patients (Table 3).

1. Nineteen patients (5.6\%) were admitted to ICU for organ failure during their hospital stay - ten ( $6.5 \%)$ patients under 65 and nine (4.8\%) over 65 . Thirteen of the 19 patients admitted to ICU $(68.4 \%)$ 
required inotropic support, nine (47.4\%) required ventilatory support and one $(5.3 \%)$ required haemodialysis; of these, three $(15.8 \%)$ had multi-organ failure requiring two or more supports. There was no statistically-significant difference between younger and older cohorts in terms of organsupport requirements.

\section{Discussion}

There has been a disproportionate increase in the number of elderly people within the population over the last 50 years, with the global population aged 60 years or older projected to treble to nearly 2 billion people within the first half of this century[21]. In Europe alone, almost $30 \%$ of the population is predicted to be aged 65 or over by 2050[21]. Geriatric patients frequently undergo emergency general surgery and accrue a greater risk of postoperative complications and fatal outcomes than the general population[6, 9, 11]. It is thus highly relevant to develop the most appropriate care measures and to guide patient-centered decision-making around emergent care[22]. ACCBD is a relatively-frequent diagnosis in patients over 65 years, and while some consensus guidance exists, age is not considered to be a contraindication to operative management[2].

This snapshot audit, a collaborative non-randomized observation study of 338 consecutive patients presenting to 25 hospitals across 9 countries over the period of one month, provides unique granular 'realworld' data on the differing treatment strategies offered to elderly patients, and the disparity in outcomes between these patients and those under 65 years of age.

Snapshot audit, a form of prospective, observational, non-randomised multi-centre cohort study, is a novel methodology for prospective collaborative cohort studies that has allowed detailed, defined datasets to be accrued in line with a priori analyses stated in pre-publication, open access protocols filed with clinical trial repositories ${ }^{2-4}$. This methodology, which is superior in some respects to large retrospective registries, has been recently embraced by surgeons as a pragmatic alternative to randomized controlled trial.

The older patients presented with the burden of cardiovascular, endocrine and renal co-morbidity far in excess of that seen in younger patients, as evidenced by a significantly higher CCI[14-16]. While a direct correlation has yet to be studied between aaCCl and morbidity and mortality following emergency cholecystectomy, strong evidence exists supporting aaCCl as a robust predictor of poor outcomes following elective gynecologic, oncologic gastrointestinal and emergency orthopedic surgery. Indeed, the observed case fatality rate in this study was $2.2 \%$ in patients over 65 years while a post-operative complication occurred in $18 \%$.

Notably, recovery following acute illness and particularly surgical intervention may be prolonged in the elderly population, as a consequence of a loss of strength, mobility, and functional capacity[23-26]. Frailty may manifest phenotypically as decline in lean body mass, strength, endurance, balance, walking performance and low activity; patients who have three or more of the five features of slowness, 
weakness, exhaustion, weight loss and low physical activity are deemed frail, while those who have none of the features are non-frail. Patients who display one or two of the five features are "pre-frail". The Canadian Study of Health and Aging (CSHA) Frailty Index[25] is based on comprehensive geriatric assessment, calculated by counting the number of deficits present in an individual, divided by the total number of deficits measured. The deficits encompass co-morbidities, physical and cognitive impairments, psychosocial risk factors and common geriatric syndromes. While our snapshot audit was not set up to specifically capture metrics of frailty, post-operative length of in-patient hospital stay was found to be significantly longer in our patients over 65 , with ongoing morbidity was reported in $13.5 \%$ of these patients and $13 \%$ (versus $2 \%$ in patients under 65 ) required discharge from the hospital to a rehabilitation facility before returning home.

\section{Conclusion}

Although this study is limited by its intention and design as a descriptive study, granular 'real world' data illustrate the challenges faced by surgeons treating elderly patients. Given the increase in morbidity, mortality, length of post-operative hospital stay and the need for rehabilitation, routine use of prognostic tools (such as p-POSSUM or aaCCl in the case of morbidity and mortality) and frailty indices in mitigating risk, informing consent and in anticipating the requirement for enhanced supports in this patient cohort.

\section{Tables}

Table 1 - Demographics (Age, Sex) of patients included in snapshot audit 


\begin{tabular}{|c|c|c|c|c|}
\hline \multirow[b]{2}{*}{ Sex } & & \multicolumn{3}{|c|}{ over $65^{\star}(p=0.059)$} \\
\hline & & under 65 years & over 65 years & Total \\
\hline \multirow[t]{2}{*}{ Male } & Observed & 62 & 94 & 156 \\
\hline & $\%$ within column & $40.5 \%$ & $50.8 \%$ & $46.2 \%$ \\
\hline \multirow[t]{2}{*}{ Female } & Observed & 91 & 91 & 182 \\
\hline & $\%$ within column & $59.5 \%$ & $49.2 \%$ & $53.8 \%$ \\
\hline \multirow[t]{2}{*}{ Total } & Observed & 153 & 185 & 338 \\
\hline & $\%$ within column & $100.0 \%$ & $100.0 \%$ & $100.0 \%$ \\
\hline
\end{tabular}

\begin{tabular}{|c|c|c|c|c|}
\hline \multirow[b]{2}{*}{ Primary diagnosis } & & \multicolumn{3}{|c|}{ over $65^{*}(p=0.065)$} \\
\hline & & under 65 years & over 65 years & Total \\
\hline \multirow[t]{2}{*}{ Cholecystitis } & Observed & 68 & 86 & 154 \\
\hline & $\%$ within column & $44.4 \%$ & $46.5 \%$ & $45.6 \%$ \\
\hline \multirow[t]{2}{*}{ Pancreatitis } & Observed & 40 & 31 & 71 \\
\hline & $\%$ within column & $26.1 \%$ & $16.8 \%$ & $21.0 \%$ \\
\hline \multirow[t]{2}{*}{ Cholangitis } & Observed & 16 & 31 & 47 \\
\hline & $\%$ within column & $10.5 \%$ & $16.8 \%$ & $13.9 \%$ \\
\hline \multirow[t]{2}{*}{ CBD stone } & Observed & 25 & 36 & 61 \\
\hline & $\%$ within column & $16.3 \%$ & $19.5 \%$ & $18.0 \%$ \\
\hline \multirow[t]{2}{*}{ fistula } & Observed & 4 & 1 & 5 \\
\hline & $\%$ within column & $2.6 \%$ & $0.5 \%$ & $1.5 \%$ \\
\hline \multirow[t]{2}{*}{ Total } & Observed & 153 & 185 & 338 \\
\hline & $\%$ within column & $100.0 \%$ & $100.0 \%$ & $100.0 \%$ \\
\hline
\end{tabular}

Table 2 - Surgical intervention in patients over and under 65 years 


\begin{tabular}{lllll}
\hline \multirow{2}{*}{ Surgicalintervention } & & \multicolumn{2}{l}{ over $65^{*}(\mathbf{p}<0.001)$} & \\
\hline No & & under 65 years & over 65 years & Total \\
\hline & Observed & 54 & 115 & 169 \\
\hline Yes & \% within column & $35.3 \%$ & $62.2 \%$ & $50.0 \%$ \\
\hline & Observed & 99 & 70 & 169 \\
\hline Total & \% within column & $64.7 \%$ & $37.8 \%$ & $50.0 \%$ \\
\hline & Observed & 153 & 185 & 338 \\
\hline & \% within column & $100.0 \%$ & $100.0 \%$ & $100.0 \%$
\end{tabular}

Table 3 - Post-operative complications in patients under and over 65 years

\begin{tabular}{llll}
\hline \multicolumn{4}{l}{ over $65(\mathrm{p}=\mathbf{0 . 1 0 2})$} \\
\hline Post-operative Complications & under 65 years & over 65 years & Total \\
\hline Abscess & 3 & 4 & 7 \\
\hline Bile duct injury & 2 & 6 & 8 \\
\hline Haemorrhage & 0 & 1 & 1 \\
\hline Wound infection & 3 & 2 & 5 \\
\hline Enterotomy & 1 & 0 & 1 \\
\hline Total & 9 & 13 & 22
\end{tabular}

Table 4 - Time from admission to endoscopy, interventional radiology and surgical intervention for patients under and over 65 years 


\begin{tabular}{lllllll}
\hline & Group & N & Mean & Median & SD & P \\
\hline $\begin{array}{l}\text { Admission to } \\
\text { Endoscopy }\end{array}$ & $\begin{array}{l}\text { under } 65 \\
\text { years }\end{array}$ & 42 & 5.00 & 4.00 & 6.71 & \\
\hline (days) & $\begin{array}{l}\text { over 65 } \\
\text { years }\end{array}$ & 62 & 9.27 & 6.50 & 11.2 & 0.009 \\
\hline Admission to IR & $\begin{array}{l}\text { under 65 } \\
\text { years }\end{array}$ & 17 & 3.65 & 2.00 & 4.33 & \\
\hline $\begin{array}{l}\text { over 65 } \\
\text { years }\end{array}$ & 18 & 4.11 & 2.00 & 12.3 & 0.738 \\
\hline $\begin{array}{l}\text { Admission to } \\
\text { Surgery }\end{array}$ & $\begin{array}{l}\text { under 65 } \\
\text { years }\end{array}$ & 96 & 10.41 & 2.00 & 21.96 & \\
\hline $\begin{array}{l}\text { over 65 } \\
\text { years }\end{array}$ & 67 & 7.25 & 1.00 & 19.4 & 0.04 \\
\hline
\end{tabular}

Table 5 - Post-acute disposition of elderly patients, statified by surgical intervention versus nonoperative management.

\begin{tabular}{clllll}
\hline & & \multicolumn{3}{l}{ Surgicalintervention } & \\
\hline over 65 & Disposition & & No & Yes & Total \\
\hline \multirow{2}{*}{ Home } & Observed & 94 & 63 & 157 \\
\hline & \% within column & $81.7 \%$ & $90.0 \%$ & $84.9 \%$ \\
\hline RIP & Observed & 3 & 1 & 4 \\
\hline & \% within column & $2.6 \%$ & $1.4 \%$ & $2.2 \%$ \\
\hline Convalescence & Observed & 18 & 6 & 24 \\
\hline & \% within column & $15.7 \%$ & $8.6 \%$ & $13.0 \%$ \\
\hline Total & Observed & 115 & 70 & 185 \\
\hline & \% within column & $100.0 \%$ & $100.0 \%$ & $100.0 \%$ \\
\hline
\end{tabular}

\section{Abbreviations}


AAST - American Association for the Surgery of Trauma

aaCCl - age-adjusted Charlson co-morbidity index

ACCBD - Acute Complicated Calculous Biliary Disease

CHSA - Canadian Study of Health and Aging Frailty Index

ERCP - Endoscopic Retrograde Cholangiopancreatography

ESTES - European Society of Trauma and Emergency Surgery

ICU - Intensive Care Unit

IQR - inter-quartile range

p-POSSUM - Portsmouth Physiological and Operative Severity Score for the Enumeration of Mortality and Morbidity

\section{Declarations}

\section{Ethics approval and consent to participate}

All 25 participating centres (see Supplemental Table of Collaborators) provided local institutional review board approval or equivalent as a requirement of registration. No patient consent was sought since the current study was purely observational and did not change the medical course of any patient. All data were irrevocably de-identified at source when uploaded to the secure study database.

\section{Consent for publication}

All authors consent to publication. This manuscript is not currently under review elsewhere.

\section{Availability of data and material}

The datasets used and/or analysed during the current study are available from the corresponding author on reasonable request.

\section{Competing interests}

Prof Shahin Mohseni is a member of the editorial board (Associate Editor) of BMC Surgery

\section{Funding}

No funding

\section{Authors' contributions}


GAB, AEG, YC and SM conceived the study, analysed the data and wrote the manuscript. All study collaborators sought local ethics approval, provided the anonymous patient-level data and per protocol are listed as PubMed-indexed authors.

\section{Acknowledgements}

The authors would like to thank the European Society for Trauma and Emergency Surgery for facilitating this collaborative effort. In particular, thanks are due to Dr Jorge Pereira, Dr Michael Sugrue and Prof Andreas Shamiyeh of the Emergency Surgery Committee of ESTES.

\section{References}

1. Garcés-Albir M, Martín-Gorgojo V, Perdomo R, Molina-Rodríguez J, Muñoz-Forner E, Dorcaratto D, et al. Acute cholecystitis in elderly and high-risk surgical patients: is percutaneous cholecystostomy preferable to emergency cholecystectomy? J Gastrointest Surg Official J Soc Surg Alimentary Tract. 2019.

2. Pisano M, Ceresoli M, Cimbanassi S, Gurusamy K, Coccolini F, Borzellino G, et al. 2017 WSES and SICG guidelines on acute calcolous cholecystitis in elderly population. World J Emerg Surg. 2019;14:10.

3. Desserud K, Veen T, Søreide K. Emergency general surgery in the geriatric patient. Brit J Surg. 2016;103:e52-61.

4. Furlong H, Bass G, Breathnach O, O'Neill B, Leen E, Walsh TN. Targeting therapy for esophageal cancer in patients aged 70 and over. J Geriatr Oncol. 2013;4:107-13.

5. Chambers D. Anaesthesia in the elderly. Anaesth Intensive Care Medicine. 2019.

6. Lidsky M, Thacker J, Lagoo-Deenadayalan S, Scarborough J. Advanced age is an independent predictor for increased morbidity and mortality after emergent surgery for diverticulitis. Surgery. 2012;152:465-72.

7. Gaitanidis A, Mikdad S, Breen K, Kongkaewpaisan N, Mendoza A, Saillant N, et al. The Emergency Surgery Score (ESS) accurately predicts outcomes in elderly patients undergoing emergency general surgery. Am J Surg. 2020.

8. Ikeuchi $\mathrm{H}$, Uchino $\mathrm{M}$, Matsuoka $\mathrm{H}$, Bando $\mathrm{T}$, Hirata $\mathrm{A}$, Takesue $\mathrm{Y}$, et al. Prognosis following emergency surgery for ulcerative colitis in elderly patients. Surg Today. 2014;44:39-43.

9. McKay A, Katz A, Lipschitz J. A population-based analysis of the morbidity and mortality of gallbladder surgery in the elderly. Surg Endosc. 2013;27:2398-406.

10. Kristjansson S, Rønning B, Hurria A, Skovlund E, Jordhøy M, Nesbakken A, et al. A comparison of two pre-operative frailty measures in older surgical cancer patients. J Geriatr Oncol. 2012;3:1-7. 
11. Khan S, Chua H, Hirubalan P, Karthekeyan R, Kothandan H. Association between frailty, cerebral oxygenation and adverse post-operative outcomes in elderly patients undergoing non-cardiac surgery: An observational pilot study. Indian J Anaesth. 2016;60:102-7.

12. Lin H, Watts J, Peel N, Hubbard R. Frailty and post-operative outcomes in older surgical patients: a systematic review. Bmc Geriatr. 2016;16:157.

13. Richards S, Frizelle F, Geddes J, Eglinton T, Hampton M. Frailty in surgical patients. Int J Colorectal Dis. 2018;33:1657-66.

14. Lin J-X, Huang Y-Q, Xie J-W, Wang J, Lu J, Chen Q-Y, et al. Age-adjusted Charlson Comorbidity Index (ACCl) is a significant factor for predicting survival after radical gastrectomy in patients with gastric cancer. Bmc Surg. 2019;19:53.

15. Kahl A, Bois A du, Harter P, Prader S, Schneider S, Heitz F, et al. Prognostic Value of the Age-Adjusted Charlson Comorbidity Index (ACCI) on Short- and Long-Term Outcome in Patients with Advanced Primary Epithelial Ovarian Cancer. Ann Surg Oncol. 2017;24:3692-9.

16. St-Louis E, lqbal S, Feldman LS, Sudarshan M, Deckelbaum DL, Razek TS, et al. Using the ageadjusted Charlson comorbidity index to predict outcomes in emergency general surgery. J Trauma Acute Care. 2015;78:318-23.

17. Cao Y, Bass GA, Ahl R, Pourlotfi A, Geijer H, Montgomery S, et al. The importance of P-POSSUM for predicting mortality after emergency laparotomy in geriatric patients. BMC Medical Informatics and Decision Making. 2020;:Article in Press.

18. Bass GA, Gillis AE, Cao Y, Mohseni S, Group ES for T and ES (ESTES) CS. A Critical View of Safety -Self-reported and actual adherence to the Tokyo Guidelines in the European Snapshot Audit on Complicated Calculous Biliary Disease. BJS Open. :Article in Press.

19. Bass GA, Gillis AE, Cao Y, Mohseni S, Group ES for T and ES (ESTES) CS. Patterns of prevalence and contemporary clinical management strategies in complicated acute biliary calculous disease - an ESTES 'snapshot audit' of practice, to European Journal of Trauma and Emergency Surgery. EJTES. 2020;:Article in Press.

20. Bass G, Gilani SNS, Walsh TN. Validating the $5 \mathrm{Fs}$ mnemonic for cholelithiasis: time to include family history. Postgrad Med J. 2013;89:638.

21. World Population Ageing: 1950-2050.

22. Dahlberg J, Dahl V, Forde R, Pedersen R. Lack of informed consent for surgical procedures by elderly patients with inability to consent: a retrospective chart review from an academic medical center in Norway. Patient Saf Surg. 2019;13:24. 
23. Theou O, Rockwood M, Mitnitski A, Rockwood K. Disability and co-morbidity in relation to frailty: How much do they overlap? Arch Gerontol Geriat. 2012;55:e1-8.

24. Rockwood K, Song X, MacKnight C, Bergman H, Hogan D, McDowell I, et al. A global clinical measure of fitness and frailty in elderly people. Can Med Assoc J. 2005;173:489-95.

25. Evaluation of a frailty index based on a comprehensive geriatric assessment in a population based study of elderly Canadians. Cochrane Central Register Control Trials Central. 2018;2007.

26. Launay-Savary M, Mathonnet M, Theissen A, Ostermann S, Raynaud-Simon A, Slim K, et al. Are enhanced recovery programs in colorectal surgery feasible and useful in the elderly? A systematic review of the literature. J Visc Surg. 2017;154:29-35.

\section{Figures}
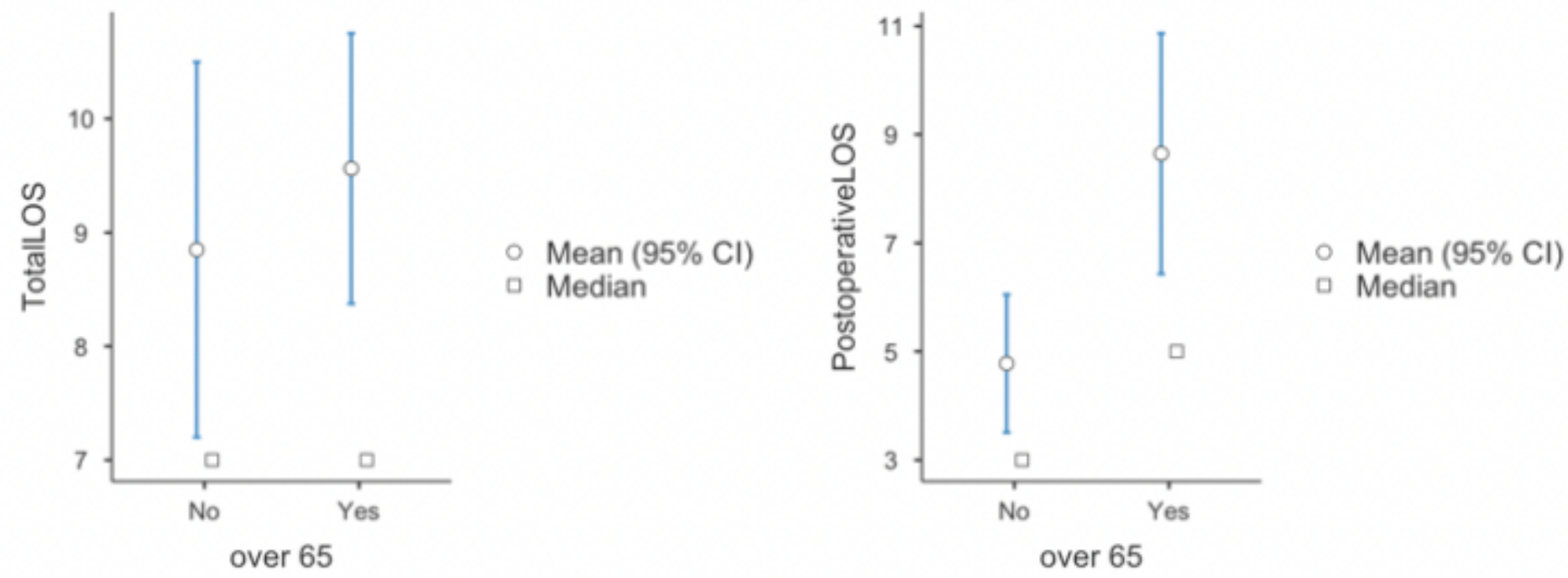

\section{Figure 1}

Total and Post operative length of stay for patients under and over the age of 65 years. 